\title{
On an Equivariant Version of the Zeta Function of a Transformation
}

\author{
S. M. Gusein-Zade ${ }^{1}$ - I. Luengo ${ }^{2,3}$. \\ A. Melle-Hernández ${ }^{2,3}$
}

Received: 17 December 2014 / Accepted: 4 April 2015 / Published online: 28 April 2015

(C) Institute for Mathematical Sciences (IMS), Stony Brook University, NY 2015

\begin{abstract}
Earlier the authors offered an equivariant version of the classical monodromy zeta function of a $G$-invariant function germ with a finite group $G$ as a power series with the coefficients from the Burnside ring of the group $G$ tensored by the field of rational numbers. One of the main ingredients of the definition was the definition of the equivariant Lefschetz number of a $G$-equivariant transformation given by W. Lück and J. Rosenberg. Here we use another approach to a definition of the equivariant Lefschetz number of a transformation and describe the corresponding notions of the equivariant zeta function. This zeta-function is a power series with the coefficients from the Burnside ring itself. We give an A'Campo type formula for the equivariant monodromy zeta function of a function germ in terms of a resolution. Finally we discuss orbifold versions of the Lefschetz number and of the monodromy zeta function corresponding to the two equivariant ones.
\end{abstract}

Keywords Equivariant Lefschetz numbers $\cdot$ Zeta functions $\cdot$ Burnside ring

S. M. Gusein-Zade partially supported by the Grants RFBR-13-01-00755 and NSh-5138.2014.1. I. Luengo and A. Melle-Hernández partially supported by the Spanish Grant MTM2013-45710-C2-2-P.

$凶$ S. M. Gusein-Zade

sabir@mccme.ru

I. Luengo

iluengo@ucm.es

A. Melle-Hernández

amelle@ucm.es

1 Faculty of Mathematics and Mechanics, GSP-1, Moscow State University, Moscow 119991, Russia

2 Present Address: ICMAT-Institute of Mathematical Sciences, Madrid, Spain

3 Faculty of Mathematical Sciences, Complutense University of Madrid, 28040 Madrid, Spain 
Mathematics Subject Classification $32 \mathrm{~S} 05 \cdot 32 \mathrm{~S} 50 \cdot 57 \mathrm{R} 91 \cdot 58 \mathrm{~K} 10$

\section{Introduction}

Many topological invariants have equivariant versions for spaces with actions of a group $G$, say, a finite one. For example, in Verdier (1973), an equivariant version of the Euler characteristic is an element of the Grothendieck ring of $\mathbb{Z}[G]$ - or $\mathbb{Q}[G]$ modules. In tom Dieck (1979, Section 5.4) it is defined as an element of the Burnside ring of the group $G$ (that is of the Grothendieck ring $K_{0}$ (f. $G$-s.) of finite $G$-sets). Applying these concepts to the Milnor fibre, one gets an equivariant version of the Milnor number of a $G$-invariant function-germ. For example, in Wall (1980) it is an element of the ring of virtual representations of the group $G$.

An important invariant of a germ of a holomorphic function (on $\left(\mathbb{C}^{n}, 0\right)$ or on a germ of a complex analytic variety) is its monodromy and its corresponding zeta function, see e.g. Arnold et al. (1988). It is defined as the zeta function of the classical monodromy transformation on the Milnor fibre. A number of statements have natural formulations in terms of monodromy zeta functions. As an example one can indicate the wellknown monodromy conjecture: see, e.g., Denef and Loeser (1992). The monodromy zeta function is connected with a number of other invariants, topological and analytic ones. For example, in Gusein-Zade et al. (1999), it was shown that, for an irreducible plane curve singularity, the monodromy zeta function of the corresponding functiongerm coincides with the Poincaré series of the natural filtration on the local ring defined by the curve valuation. There are generalizations of this fact to some other situations [(see, e.g., a survey in Gusein-Zade (2010)]. In all these cases one has no intrinsic explanation of the relation. The relation is obtained by independent computation of the right and left hand sides of it in the same terms and comparison of the obtained results.

Generalizations of relations of this sort to equivariant settings could help to understand the general framework. For example, in Ebeling and Gusein-Zade (2012b) an equivariant version of a relation obtained earlier gave a better understanding of the role of the Saito duality in it. This leads to the desire to define equivariant analogues of monodromy zeta functions and of the Poincaré series of filtrations. These problem is not trivial and equivariant analogues are not unique. Moreover, up to now there were no definitions of equivariant analogues of monodromy zeta functions and of the Poincaré series which were elements of the same rings. For example, in Campillo et al. (2007, 2013), there were offered different approaches to equivariant Poincaré series. In Campillo et al. (2007) it is a power series with coefficients from the ring of one-dimensional representation of a group. In Campillo et al. (2013) it is an element of the Grothendieck ring of "locally finite" $G$-sets with an additional structure. In Gusein-Zade et al. (2008), there was given an equivariant version of the monodromy zeta function as a power series with the coefficients from $K_{0}$ (f. $G$-s.) $\otimes \mathbb{Q}$. The fact that it was defined only after tensoring by the field $\mathbb{Q}$ of rational numbers makes it less reasonable, in particular, to compare it with the equivariant versions of the Poincaré series which were defined over integers. In Gusein-Zade (2013) an equivariant version of the monodromy zeta function was defined as an element of a generalization of the Burnside ring different from that in Campillo et al. (2013). Just recently one gave a 
definition of an equivariant version of the Poincare series as a power series with the coefficients from the Burnside ring of the group: Campillo et al. (2015). (In that paper initially the Poincaré series is defined as a power series with the coefficients from a certain modification of the Burnside ring. A simple reductions sends this modification to the usual Burnside ring.)

One of the main ingredients of the definition of the equivariant version of the monodromy zeta function in Gusein-Zade et al. (2008) was the definition of the equivariant Lefschetz number of a transformation from Lück and Rosenberg (2003). The definition from Lück and Rosenberg (2003) is rather natural. Moreover, one can say that it is the only possible definition possessing some reasonable properties. However the fact that it leads to a "non-integer" definition of the (monodromy) zeta function gives a hint that this definition is not absolutely adequate to this purpose.

There is certain freedom in a definition of an equivariant version of the Lefschetz number of a transformation connected with the question whether it should count the fixed points of the transformation or the fixed G-orbits of it. Here we use the second approach to the definition of the equivariant version of the Lefschetz number. This definition was introduced in Dzedzej (2001). We describe the corresponding equivariant version of the zeta function of a transformation. This zeta-function is a power series with the coefficients from the ring $K_{0}$ (f. $G$-s.). We give an A'Campo type formula for the equivariant monodromy zeta function of a function germ in terms of a resolution.

Difficulties to compare equivariant versions of monodromy zeta functions and of the Poincaré series being elements of different nature (e.g. those described above) leads to the idea to compare their "integer valued reductions". These reductions can be made with the help of the usual Euler characteristic and also with the help of the orbifold Euler characteristic. In the light of this, we also discuss possible orbifold versions of the zeta function of a transformation.

Remark The defined equivariant version of the zeta function is not a new invariant in the sense that it cannot distinguish more transformations than existing ones. In particular, it is expressed in terms of equivariant Lefschetz numbers of iterates defined earlier. The same holds for the usual (non-equivariant) zeta function of a transformation. However, it appears to be better adapted to a number of problems. (One more example: the well-known monodromy conjecture on poles of the topological Igusa zeta function (see, e.g., Denef and Loeser 1992) is also formulated in terms of the monodromy zeta function.) To find equivariant analogues of these problems, one would need to have an equivariant generalization of the usual zeta function of a transformation. Moreover, there are other indices of equivariant transformations which are more fine invariants than the monodromy zeta function. For example, equivariant generalizations of Dold's indices of iterates defined in Crabb (2007) are of this sort: our equivariant version of the zeta function can be expressed through them (private communication by M. C. Crabb).

\section{Burnside Ring and the Equivariant Euler Characteristic}

A finite $G$-set is a finite set with an action (say a left one) of the group $G$. Isomorphism classes of irreducible $G$-sets (i.e. those which consist of exactly one orbit) are in one-toone correspondence with the set $\operatorname{Consub}(G)$ of conjugacy classes of subgroups of $G$ : 
to the conjugacy class containing a subgroup $H \subset G$ one associates the isomorphism class $[G / H]$ of the $G$-set $G / H$. The Grothendieck ring $K_{0}$ (f. $G$-s.) of finite $G$-sets (also called the Burnside ring of $G$ ) is the group generated by isomorphism classes of finite $G$-sets with the relation $[A \coprod B]=[A]+[B]$ and with the multiplication defined by the cartesian product. As an abelian group $K_{0}$ (f. $G$-s.) is freely generated by the isomorphism classes $[G / H]$ of irreducible $G$-sets. The element 1 in the ring $K_{0}$ (f. $G$-s.) is represented by the $G$-set consisting of one point (with the trivial $G$ action).

Recall that given a subgroup $H$ of $G$ there are two natural maps $\operatorname{Res}_{H}^{G}$ : $K_{0}$ (f. $G$-sets) $\rightarrow K_{0}$ (f. $H$-sets) and $\mathrm{Ind}_{H}^{G}: K_{0}$ (f. $H$-sets) $\rightarrow K_{0}$ (f. $G$-sets). The restriction map $\operatorname{Res}_{H}^{G}$ sends a $G$-set X to the same set considered with the $H$-action. The induction map $\operatorname{Ind}_{H}^{G}$ sends an $H$-set $X$ to the product $G \times X$ factorized by the natural equivalence: $\left(g_{1}, x_{1}\right) \sim\left(g_{2}, x_{2}\right)$ if there exists $g \in H$ such that $g_{2}=g_{1} g$, $x_{2}=g^{-1} x_{1}$ with the natural (left) $G$-action. The induction map $\operatorname{Ind}_{H}^{G}$ sends the class $\left[H / H^{\prime}\right]\left(H^{\prime}\right.$ is a subgroup of $\left.H\right)$ to the class $\left[G / H^{\prime}\right]$. Both maps are group homomorphisms, however the induction map $\operatorname{Ind}_{H}^{G}$ is not a ring homomorphism.

In some places, say, in tom Dieck (1979), Lück and Rosenberg (2003) and GuseinZade et al. (2008), the equivariant Euler characteristic of a $G$-space is considered as an element of the Grothendieck ring $K_{0}$ (f. $G$-s.). For a relatively good $G$-space $X$ (say, for a quasiprojective variety) the equivariant Euler characteristic $\chi^{G}(X) \in K_{0}$ (f. $G$-s.) can be defined in the following way. For a point $x \in X$, let $G_{x}=\{g \in G: g \cdot x=x\}$ be the isotropy subgroup of the point $x$. For a conjugacy class $\mathcal{H} \in \operatorname{Consub}(G)$, set $X^{\mathcal{H}}=\{x \in X: x$ is a fixed point of a subgroup $H \in \mathcal{H}\}$ and let $X^{(\mathcal{H})}=\{x \in X$ : $G_{x} \in \mathcal{H}$ \} be the set of points with the isotropy subgroups from $\mathcal{H}$. One can see that in the natural sense $X^{(\mathcal{H})}=X^{\mathcal{H}} \backslash X^{>\mathcal{H}}$, where $X^{>\mathcal{H}}=\bigcup_{\mathcal{H}^{\prime}>\mathcal{H}} X^{\mathcal{H}^{\prime}}$.

Then the equivariant Euler characteristic of the $G$-space $X$ is defined as

$$
\chi^{G}(X):=\sum_{\mathcal{H} \in \operatorname{Consub}(G)} \frac{\chi\left(X^{(\mathcal{H})}\right)|H|}{|G|}[G / H]=\sum_{\mathcal{H} \in \operatorname{Consub}(G)} \chi\left(X^{(\mathcal{H})} / G\right)[G / H]
$$

where $H$ is a representative of the conjugacy class $\mathcal{H}$.

Remark Here we use the additive Euler characteristic $\chi(\cdot)$, i.e. the alternating sum of the ranks of the cohomology groups with compact support. For a complex analytic variety this Euler characteristic is equal to the alternating sum of the ranks of the usual cohomology groups.

Definition A pre- $\lambda$ ring structure on a commutative ring $R$ is an additive to multiplicative group homomorphism $\lambda_{T}: R \rightarrow 1+T \cdot R[[T]]$, that is $\lambda_{T}(m+n)=\lambda_{T}(m) \lambda_{T}(n)$, such that $\lambda_{T}(m)=1+m T\left(\bmod T^{2}\right)$.

A pre- $\lambda$ ring homomorphism is a ring homomorphism between pre- $\lambda$ rings which commutes with the pre- $\lambda$ ring structures.

The Grothendieck ring $K_{0}$ (f. $G$-s.) has a natural pre- $\lambda$-ring structure defined by the series

$$
\sigma_{X}(t)=1+[X] t+\left[S^{2} X\right] t^{2}+\left[S^{3} X\right] t^{3}+\cdots
$$


where $S^{k} X=X^{k} / S_{k}$ is the $k$-th symmetric power of the $G$-set $X$ with the natural $G$-action. This pre- $\lambda$-ring structure induces a power structure over the Grothendieck ring $K_{0}$ (f. $G$-s.): see Gusein-Zade et al. (2006). This means that for a power series $A(t) \in 1+t \cdot K_{0}$ (f. $G$-s.) $[[t]]$ and $m \in K_{0}$ (f. $G$-s.) there is defined a series $(A(t))^{m} \in$ $1+t \cdot K_{0}$ (f. $G$-s.) $[[t]]$ so that all the properties of the exponential function hold. In these notations $\sigma_{X}(t)=(1-t)^{-[X]}$. The geometric description of the natural power structure over the Grothendieck ring of quasiprojective varieties given in Gusein-Zade et al. (2006) using graded spaces is also valid for the power structure over $K_{0}$ (f. $G$-s.) as well.

Some examples of computation of the series $(1-t)^{-[G / H]}$ for $G$ being the cyclic group $\mathbb{Z}_{6}$ of order 6 and the group $\mathcal{S}_{3}$ of permutations on three elements can be found in Gusein-Zade et al. (2008) (with some misprints). For example

$$
\begin{aligned}
(1-t)^{-\left[\mathcal{S}_{3} /\langle e\rangle\right]}= & \frac{1}{1-t^{6}}[1]+\frac{t^{3}}{\left(1-t^{3}\right)\left(1-t^{6}\right)}\left[\mathcal{S}_{3} / \mathbb{Z}_{3}\right] \\
& +\frac{3 t^{2}}{\left(1-t^{2}\right)^{2}\left(1-t^{6}\right)}\left[\mathcal{S}_{3} / \mathbb{Z}_{2}\right] \\
& +\frac{t\left(1+4 t^{2}+t^{3}+4 t^{4}-2 t^{5}+3 t^{6}+t^{7}\right)}{\left(1-t^{2}\right)^{2}\left(1-t^{3}\right)\left(1-t^{6}\right)(1-t)^{2}}\left[\mathcal{S}_{3} /\langle e\rangle\right]
\end{aligned}
$$

There is a natural homomorphism from the Grothendieck ring $K_{0}$ (f. $G$-s.) to the ring $R(G)$ of virtual representations of the group $G$ which sends the class $[G / H] \in$ $K_{0}$ (f. $G$-s.) to the representation $i_{H}^{G}\left[1_{H}\right]$ induced from the trivial one-dimensional representation $1_{H}$ of the subgroup $H$. (A virtual representation of the group $G$ is an element of the Grothendieck ring of representations, i.e. a formal difference of two representations.) This homomorphism is a homomorphism of pre- $\lambda$-rings (Knutson 1973).

Let us show that for any subgroup $H$ of $G$ the series $(1-t)^{-[G / H]}$ represents a rational function with the denominator equal to a product of the binomials of the form $\left(1-t^{m}\right), m \in \mathbb{Z}_{\geq 1}$. Since irreducible $G$-sets are in one-to-one correspondence with the set $\operatorname{Consub}(G)$ of conjugacy classes of subgroups of $G$ and $K_{0}($ f. $G$-s.) is freely generated by isomorphism classes $[G / H]$ of irreducible $G$-sets then

$$
(1-t)^{-[G / H]}=\sum_{\mathcal{F} \in \operatorname{Consub}(G)} \mathcal{A}_{H, \mathcal{F}}(t)[G / F]
$$

where $F$ is a representative of the conjugacy class $\mathcal{F}$ and $\mathcal{A}_{H, \mathcal{F}}(t) \in \mathbb{Z}[[t]]$.

Let $\mathcal{F}$ be a conjugacy class of subgroups of $G$ and let $F$ be a representative of it. The subgroup $F$ acts on the $G$-space $G / H$. Let $F \backslash G / H$ be the quotient of $G / H$ by this action and let $p: G / H \rightarrow F \backslash G / H$ be the quotient map. For $m=1,2, \ldots$, let $Y_{m}$ be the set of points of $F \backslash G / H$ with $m$ preimages in $G / H$ and let $\ell_{m}^{\mathcal{F}}=\left|Y_{m}\right|$. (The numbers $\ell_{m}^{\mathcal{F}}$ depend only on the conjugacy class $\mathcal{F}$.) For an abelian $G, \ell_{m}^{\mathcal{F}}$ is different from zero if and only if $m=\frac{|F|}{|F \cap H|}$ and in this case $\ell_{m}^{\mathcal{F}}=|G| /|F+H|$.

For conjugacy classes $\mathcal{F}$ and $\mathcal{F}^{\prime}$ from $\operatorname{Consub}(G)$, let $F$ and $F^{\prime}$ be their representatives, and let $r_{\mathcal{F}^{\prime}, \mathcal{F}}$ be the number of fixed points of the group $F$ on $G / F^{\prime}$. The 
integer $r_{\mathcal{F}^{\prime}, \mathcal{F}}$ is different from zero if and only if $\mathcal{F}^{\prime} \geq \mathcal{F}$ (i.e. there exist representatives $F^{\prime}$ of $F$ of them such that $F^{\prime} \supset F$. For an abelian $G$ and for $\mathcal{F}^{\prime} \geq \mathcal{F}$, one has $r_{\mathcal{F}^{\prime}, \mathcal{F}}=\left|G / F^{\prime}\right|$. For a non-abelian group the equation is more involved and $r_{\mathcal{F}^{\prime}, \mathcal{F}}$ depends on $\mathcal{F}$ as well.

Lemma 1 For $\mathcal{F} \in \operatorname{Consub}(G)$ one has

$$
\prod_{m \geq 1}\left(1-t^{m}\right)^{-\ell_{m}^{\mathcal{F}}}=\sum_{\mathcal{F}^{\prime} \in \operatorname{Consub}(G)} r_{\mathcal{F}^{\prime}, \mathcal{F}} \mathcal{A}_{H, \mathcal{F}^{\prime}}(t)
$$

Proof Let $F$ be a representative of $\mathcal{F}$ and let us count fixed points of the subgroup $F$ in the left hand side and right hand side of (2).

For a finite set $X$ an element of $\coprod_{k \geq 0} S^{k} X$ can be identified with an integer valued function on $X$ with non-negative values. The corresponding element belongs to $S^{k} X$ if and only if the sum of all the values of the function is equal to $k$. An element of $\bigsqcup_{k \geq 0} S^{k}[G / H]$ is fixed with respect to $F$ if and only if the corresponding function is invariant with respect to the $F$-action on $G / H$. Such a function can be identified with a function on $F \backslash G / H$. A function on $F \backslash G / H$ can be also considered as the direct sum of functions on the subset $Y_{S}$ defined above. The generating series for the number of functions on $Y_{s}$ (i.e. the series $\left.\sum_{k \geq 0}\left|S^{k} Y_{s}\right| t^{k}\right)$ is $(1-t)^{-\left|Y_{s}\right|}=(1-t)^{-\ell_{s}^{\mathcal{F}}}$. Each function on $Y_{S}$ with the sum of values equal to $k$ lifts to an $H$ invariant function on $G / H$ with the sum of the values equal to $k s$. Therefore the generating series for $F$-invariants functions on $p^{-1}\left(Y_{S}\right)$ is $\left(1-t^{s}\right)^{-\ell_{s}^{\mathcal{F}}}$. The generating series for all $F$-invariants functions on $G / H$ is the product of those for $p^{-1}\left(Y_{S}\right)$. This is the left hand side of (3).

The right hand side of (3) is obviously the set of fixed points of $F$ on the right hand side of (2).

Since $r_{\mathcal{F}^{\prime}, \mathcal{F}}$ is different from zero if and only if $\mathcal{F} \leq \mathcal{F}^{\prime}$ and $r_{\mathcal{F}^{\prime}, \mathcal{F}^{\prime}}$ is different from zero, the system of Eq. (3) is a triangular one (with respect to the partial order on the set of conjugacy classes of subgroups of $G$ ). Together with the fact that the denominators of the left hand side of the Eq. (3) are product of the binomials of the form $\left(1-t^{m}\right)$ this implies the following statement.

Proposition 1 For any subgroup $H$ of $G$ the series $(1-t)^{-[G / H]}$ belongs to the localization $K_{0}$ (f. $G$-s.) $[t]_{\left(\left\{1-t^{m}\right\}\right)}$ of the polynomial ring $K_{0}$ (f. $G$-s.) $[t]$ at all the elements of the form $\left(1-t^{m}\right), m \geq 1$.

The natural homomorphism from the Grothendieck ring $K_{0}$ (f. $G$-s.) to the ring $R(G)$ of virtual representations of the group $G$ sends the equivariant Euler characteristic $\chi^{G}(X)$ to the one used in Wall (1980). Since this homomorphism is, generally speaking, neither injective, no surjective, the equivariant Euler characteristic as an element in $K_{0}$ (f. $G$-s.) is a somewhat finer invariant than the one as an element of the $\operatorname{ring} R(G)$. 


\section{An Alternative Version of the Equivariant Lefschetz Number of a Map}

Let $X$ be a relatively good topological space (say, a quasiprojective complex or real variety) with a $G$-action and let $\varphi: X \rightarrow X$ be a $G$-equivariant proper map. The usual ("non-equivariant") Lefschetz number $L(\varphi)$ counts the fixed points of $\varphi$ (or rather of its generic perturbation). The equivariant version $L^{G}(\varphi)$ of the Lefschetz number from Lück and Rosenberg (2003) counts the fixed points of $\varphi$ as a (finite) $G$-set. This leads to the following equation for the equivariant Lefschetz number

$$
L^{G}(\varphi)=\sum_{\mathcal{H} \in \operatorname{Consub}(G)} \frac{L\left(\varphi_{\mid\left(X^{\mathcal{H}}, X^{>\mathcal{H}}\right)}\right)|H|}{|G|}[G / H],
$$

where $H$ is a representative of the class $\mathcal{H}$. If $\varphi$ is a $G$-homeomorphism (like the monodromy transformation, see Sect. 5), then

$$
L^{G}(\varphi)=\sum_{\mathcal{H} \in \operatorname{Consub}(G)} \frac{L\left(\varphi_{\mid X^{(\mathcal{H})}}\right)|H|}{|G|}[G / H] .
$$

Assume that one wants to count the fixed orbits of $\varphi$ (i.e. the orbits which are sent to themselves, generally speaking, not point-wise) as finite $G$-sets. This leads to the following definition of the equivariant Lefschetz number:

$$
\widetilde{L}^{G}(\varphi)=\sum_{\mathcal{H} \in \operatorname{Consub}(G)} L\left(\varphi_{\mid\left(X^{\mathcal{H}} / G, X^{>\mathcal{H}} / G\right)}\right)[G / H] .
$$

This definition was already used in Dzedzej (2001). If $\varphi$ is a $G$-homeomorphism, one has

$$
\widetilde{L}^{G}(\varphi)=\sum_{\mathcal{H} \in \operatorname{Consub}(G)} L\left(\varphi_{\mid X^{(\mathcal{H})} / G}\right)[G / H] .
$$

[It is useful to compare Eqs. (4), (5) and (6), (7) with the two parts of the equation (1).]

Example For some simplicity, let the group $G$ be abelian, let $X=(G / H) \times \mathbb{Z}_{k}=$ $\{0,1, \ldots, k-1\}, k>0$, with the natural action of the group $G$ on the first factor, and let the map $\varphi: X \rightarrow X$ be defined by

$$
\varphi(a, i)= \begin{cases}(a, i+1) & \text { for } 0 \leq i<k-1, \\ (g a, 0) & \text { for } i=k-1\end{cases}
$$

If $k>1$, then $L^{G}(\varphi)=\widetilde{L}^{G}(\varphi)=0$ since $\varphi$ has neither fixed points, no fixed orbits. The smallest $i$ for which $\widetilde{L}^{G}\left(\varphi^{i}\right) \neq 0$ is $i=k$. In this case all the $G$-orbits in $X$ are fixed by $\varphi^{k}$ and therefore $\widetilde{L}^{G}\left(\varphi^{k}\right)=k[G / H]$. On the other hand, if $g \notin H$, the map $\varphi^{k}$ has no fixed points and thus $L^{G}\left(\varphi^{k}\right)=0$. The smallest $i$ for which $L^{G}\left(\varphi^{i}\right) \neq 0$ is 
$i=\ell k$, where $\ell$ is the order of the element $g$ in the group $G / H$. In this case all the points of $X$ are fixed by $\varphi^{\ell k}$ and therefore $\widetilde{L}^{G}\left(\varphi^{\ell k}\right)=k[G / H]$.

Just in the same way as in Lück and Rosenberg (2003) one can formulate the equivariant version of the Lefschetz fixed point theorem for $\widetilde{L}^{G}(\varphi)$ (an analogue of Theorem 2.1 in Lück and Rosenberg 2003).

\section{The Zeta Function of a Transformation}

Let $\varphi: X \rightarrow X$ be as above. The usual (non-equivariant) zeta function of $\varphi$ is defined in terms of the action of $\varphi$ in the (co)homology groups of $X$ (in a way somewhat similar to the definition of the Lefschetz number). This definition is not convenient for a direct generalization to the equivariant case. It is more convenient to use the definition of the zeta function of the transformation $\varphi$ in terms of the Lefschetz numbers of the iterates of $\varphi$. One defines integers $s_{i}, i=1,2 \ldots$, recursively by the equation

$$
L\left(\varphi^{m}\right)=\sum_{i \mid m} s_{i}
$$

The number $s_{m}$ counts (with integer multiplicities) the points $x \in X$ with the $\varphi$ order equal to $m$ (i.e. $\varphi^{m}(x)=x, \varphi^{i}(x) \neq x$ for $0<i<m$ ). Together with each such point all its images under the iterates of $\varphi$ (there are exactly $m$ different ones) are of this sort. Therefore $s_{m}$ is divisible by $m$. One defines the zeta function $\zeta_{\varphi}(t)$ to be

$$
\zeta_{\varphi}(t):=\prod_{m \geq 1}\left(1-t^{m}\right)^{-s_{m} / m}
$$

Remark There are two traditions to define the zeta function of a transformation. The other one does not contain the minus sign in the exponent and therefore is the inverse to this one. Here we follow the definition from A'Campo (1975).

In the equivariant version, let $s_{m}^{G}(\varphi)$ and $\widetilde{s}_{m}^{G}(\varphi)$ be defined through $L^{G}\left(\varphi^{i}\right)$ and $\widetilde{L}^{G}\left(\varphi^{i}\right)$ respectively by the analogues of the Eq. (8)

$$
L^{G}\left(\varphi^{m}\right)=\sum_{i \mid m} s_{i}^{G}(\varphi), \quad \widetilde{L}^{G}\left(\varphi^{m}\right)=\sum_{i \mid m} \widetilde{s}_{i}^{G}(\varphi) .
$$

The elements $s_{m}^{G}(\varphi)$ and $\widetilde{s}_{m}^{G}(\varphi)$ count the points in $X$ the $\varphi$-order of which is equal to $m$ in $X$ and in $X / G$ respectively.

Example In the Example from Sect. 3

$$
\begin{aligned}
& \widetilde{s}_{i}^{G}(\varphi)=0 \text { for } i<k \quad \text { and } \widetilde{s}_{k}^{G}(\varphi)=\widetilde{L}^{G}(\varphi)=k[G / H], \\
& s_{i}^{G}(\varphi)=0 \text { for } i<\ell k \quad \text { and } s_{\ell k}^{G}(\varphi)=L^{G}(\varphi)=k[G / H] .
\end{aligned}
$$


One can see that in this case $\widetilde{s}_{k}^{G}(\varphi)$ is divisible by $k$, but $s_{\ell k}^{G}(\varphi)$ is not divisible by $\ell k$. This is a general feature. One can easily prove the following proposition.

Proposition 2 The element $\widetilde{s}_{m}^{G}(\varphi)$ in $K_{0}$ (f. $G$-s.) is divisible by $m$.

This permits to give the following definition:

Definition The equivariant zeta function of a $G$-equivariant map $\varphi: X \rightarrow X$ is the series $\widetilde{\zeta}_{\varphi}^{G}(t)$ defined by

$$
\widetilde{\zeta}_{\varphi}^{G}(t)=\prod_{m \geq 1}\left(1-t^{m}\right)^{-\widetilde{s}_{m}^{G} / m} \in 1+t \cdot K_{0}(\text { f. } G \text {-s. })[[t]],
$$

where each virtual finite $G$-set $\widetilde{s}_{m}^{G} \in K_{0}$ (f. $G$-s.) defined as above is divisible by $m$.

Remark The definition given in Gusein-Zade et al. (2008) used the elements $s_{m}^{G}$ and made sense only after tensoring by the field $\mathbb{Q}$. Here we do not need this tensoring. One reason why this is preferable consists in the fact that natural equivariant generalizations of the Poincaré series of filtrations are defined over rings which are not $\mathbb{Q}$-modules.

Applying the natural homomorphism from the Grothendieck ring $K_{0}$ (f. $G$-s.) to the ring $R(G)$ of representations of the group $G$ (see Sect. 2) one gets a reduced version of the zeta function as an element of $1+t \cdot R(G)[[t]]$. The equivariant Poincare series of filtrations defined in Campillo et al. (2007) also belongs to the set $1+t \cdot R(G)[[t]]$. (In fact the latter one even belongs to the set $1+t \cdot R_{1}(G)[[t]]$, where $R_{1}(G)$ is the subring of $R(G)$ generated by the one-dimensional representations. However, for an abelian group $G$, where the notion of the equivariant Poincaré series from Campillo et al. (2007) really makes sense, these sets coincide.)

The properties of the equivariant Lefschetz numbers and the example above imply the following propositions.

Proposition 3 Let $\varphi: X \rightarrow X$ be such that $\varphi(Y) \subset Y, \varphi(X \backslash Y) \subset X \backslash Y$ for $a$ $G$-subset $Y \subset X$. Then

$$
\widetilde{\zeta}_{\varphi}^{G}(t)=\widetilde{\zeta}_{\varphi \mid Y}^{G}(t) \cdot \widetilde{\zeta}_{\varphi \mid X \backslash Y}^{G}(t)
$$

Proposition 4 Let $X=X^{(\mathcal{H})}$ for a conjugacy class $\mathcal{H} \in \operatorname{Consub}(G)$ and let $\varphi: X \rightarrow$ $X$ be a $G$-equivariant map. Then

$$
\widetilde{\zeta}_{\varphi}^{G}(t)=\left(\zeta_{\varphi_{\mid X / G}}(t)\right)^{[G / H]}
$$

for a representative $H$ of the class $\mathcal{H}$.

Assume that a $G$-equivariant map $\varphi: X \rightarrow X$ preserves the subspaces $X^{(\mathcal{H})}$, i.e. $\varphi\left(X^{(\mathcal{H})}\right) \subset X^{(\mathcal{H})}$. In this case $\varphi_{\mid X / G}$ preserves the subspaces $X^{(\mathcal{H})} / G$. Propositions 3 and 4 imply the following statement. 
Proposition 5 Let $\varphi: X \rightarrow X$ be a G-equivariant map that preserves the subspaces $X^{(\mathcal{H})}$ for any conjugacy class $\mathcal{H} \in \operatorname{Consub}(G)$. Then

$$
\widetilde{\zeta}_{\varphi}^{G}(t)=\prod_{\mathcal{H} \in \operatorname{Consub}(G)}\left(\zeta_{\varphi_{\mid X}(\mathcal{H}) / G}(t)\right)^{[G / H]}
$$

This statement can be applied to the monodromy transformation below.

\section{The A'Campo Type Formula for the Equivariant Monodromy Zeta Function}

Let $(V, 0)$ be a germ of a purely $n$-dimensional complex analytic variety with an action of a finite group $G$ and let $f:(V, 0) \rightarrow(\mathbb{C}, 0)$ be the germ of a $G$-invariant analytic function such that Sing $V \subset f^{-1}\{0\}$. Let $M_{f}$ be the Milnor fibre of the germ $f$ at the origin: $M_{f}=\{x \in V: f(x)=\varepsilon,\|x\| \leq \delta\}$ with $0<|\varepsilon| \ll \delta$ small enough (for this definition we assume $(V, 0)$ to be embedded in the affine space $\left.\left(\mathbb{C}^{N}, 0\right)\right)$. The group $G$ acts on the Milnor fibre $M_{f}$. The classical monodromy transformation $h=h_{f}: M_{f} \rightarrow M_{f}$ is a $G$-equivariant map corresponding to the loop $\varepsilon(\tau)=\varepsilon \cdot \exp (2 \pi i \tau)$ in $\mathbb{C}$ around the origin.

Let $\pi:(X, \mathcal{D}) \rightarrow(V, 0)$ be a $G$-equivariant resolution of the germ $f$, i.e. a proper $G$-equivariant map from an $n$-dimensional $G$-manifold $X$ to $V$ such that $\pi$ is an isomorphism outside the zero level set $f^{-1}\{0\}$ of the function $f$ and, in a neighbourhood of any point $p$ of the total transform $E_{0}:=\pi^{-1}\left(f^{-1}\{0\}\right)$ of the zero-level set $f^{-1}\{0\}$ of the function $f$, there exists a local system of coordinates $z_{1}, \ldots, z_{n}$ (centred at the point $p$ ) such that $f \circ \pi\left(z_{1}, \ldots, z_{n}\right)=z_{1}^{m_{1}} z_{2}^{m_{2}} \cdots z_{n}^{m_{n}}$, with non-negative integers $m_{i}$, $i=1, \ldots, n$. (This implies that the total transform $E_{0}$ of the zero level set $f^{-1}\{0\}$ is a normal crossing divisor on $X$.) Moreover, we assume that, for each point $p \in E_{0}$, the irreducible components of $E_{0}$ at the point $p$ are invariant with respect to the isotropy group $G_{p}$ of the point $p$. (This can be achieved, if necessary, by additional blow-ups of the intersections of the components of the exceptional divisor.)

Let $S_{m}, m \geq 1$, be the set of points $p$ of the exceptional divisor $\mathcal{D}:=\pi^{-1}\{0\}$ such that, in a neighbourhood of the point $p$, one has $f \circ \pi\left(z_{1}, \ldots, z_{n}\right)=z_{1}^{m}$. For $p \in S_{m}$, let $G_{p}$ be the isotropy group of the point $p: G_{p}=\{g \in G: g p=p\}$. The group $G_{p}$ acts on the smooth germ $(X, p)$ preserving the exceptional divisor $\mathcal{D}$ locally given by $z_{1}=0$. This implies that, in a neighbourhood of the point $p$, one can suppose $G_{p}$ to act by linear transformations in coordinates $z_{1}, \ldots, z_{n}$ preserving "the normal slice" $z_{2}=\ldots=z_{n}=0$. This way one gets a linear representation of the group $G_{p}$ on this normal slice. Let $\widehat{G}_{p}$ be the kernel of this representation. One can see that $G_{p} / \widehat{G}_{p}$ is a cyclic group the order of which divides $m$.

For every ( $\widehat{H}$ subgroup of $H$, let $S_{m, H, \widehat{H}}$ be the set of points $p \in S_{m}$ such that the pair $(H, \widehat{H})$ is conjugate to the pair $\left(G_{p}, \widehat{G}_{p}\right)$, i.e. for an element $g \in G$ one has $G_{p}=g H g^{-1}$ and $\widehat{G}_{p}=g \widehat{H} g^{-1}$. 
Theorem A'Campo type formula for an equivariant monodromy zeta function

$$
\begin{aligned}
\tilde{\zeta}_{f}^{G}(t) & =\prod_{m \geq 1,(\mathcal{H}, \widehat{\mathcal{H}})}\left(1-t^{m \frac{|\widehat{H}|}{|H|}}\right)^{-\frac{|H| \chi\left(S_{m, H, \widehat{H}}\right)}{|G|}[G / \widehat{H}]} \\
& =\prod_{m \geq 1,(\mathcal{H}, \widehat{\mathcal{H}})}\left(1-t^{m \frac{|\widehat{H}|}{|H|}}\right)^{-\chi\left(S_{m, H, \widehat{H}} / G\right)[G / \widehat{H}]},
\end{aligned}
$$

where the product is over all conjugacy classes $(\mathcal{H}, \widehat{\mathcal{H}})$ of pairs of subgroups of the group $G$ such that $H / \widehat{H}$ is a cyclic group and the pair $(H, \widehat{H})$ is a representative of the conjugacy class $(\mathcal{H}, \widehat{\mathcal{H}})$.

Proof Since the resolution $\pi$ is an isomorphism outside of $f^{-1}(0)$, the Milnor fibre $M_{f}$ can be identified with its preimage $\pi^{-1}\left(M_{f}\right) \subset X$. Just like in the non-equivariant case (see A'Campo 1975; Clemens 1969) one can construct a $G$-equivariant retraction of a neighbourhood of the total transform $E_{0}$ of the zero level set $f^{-1}\{0\}$ to $E_{0}$ itself such that, outside of a neighbourhood in $E_{0}$ of all intersections of at least two irreducible components of $E_{0}$, i.e. where in local coordinates $f \circ \pi\left(z_{1}, \ldots, z_{n}\right)=z_{1}^{m}, m \geq 1$, the retraction sends a point $\left(z_{1}, z_{2}, \ldots, z_{n}\right)$ to $\left(0, z_{2}, \ldots, z_{n}\right)$. Moreover, one can construct a monodromy transformation $h=h_{f}$ which commutes with the retraction and, in a neighbourhood of a point where $f \circ \pi\left(z_{1}, \ldots, z_{n}\right)=z_{1}^{m}$, it sends a point $\left(z_{1}, z_{2}, \ldots, z_{n}\right) \in M_{f}$ to $\left(\exp \left(\frac{2 \pi i}{m}\right) z_{1}, z_{2}, \ldots, z_{n}\right) \in M_{f}, M_{f}$ being the Milnor fibre.

From Proposition 3 , it follows that the equivariant zeta function $\widetilde{\zeta}_{f}^{G}(t):=\widetilde{\zeta}_{h_{f}}^{G}(t)$ is equal to the product of the equivariant zeta functions for the monodromy transformation on the preimages of the strata $S_{m, H, \widehat{H}}$ and on neighbourhoods of the intersections of the irreducible components of $E_{0}$.

One can show that the equivariant zeta function of the monodromy transformation $h$ on a neighbourhood of an intersection of the irreducible components of $E_{0}$ is equal to one. This follows from the fact that the Milnor fibre $M_{f}$ in a neighbourhood of such an intersection can be fibred by circles and the monodromy transformation can be supposed to preserve these fibration.

To compute the equivariant zeta function of the monodromy transformation on the preimage of the stratum $S_{m, H, \widehat{H}}$ it is useful to have in mind the following property of the usual zeta function of a transformation. Let $\varphi: Y \rightarrow Y$ be such that $\varphi^{i}$ has no fixed points for $0<i<k, \varphi^{k}=i d$. Then $\widetilde{\zeta}_{\varphi}(t)=\left(1-t^{k}\right)^{-\chi(Y) / k}$. Now the mentioned above equivariant zeta function is given by Proposition 4 and is equal to

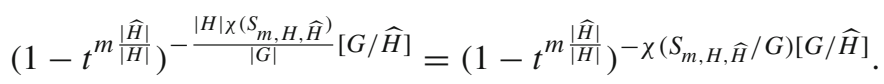

This implies (12).

Equation (12) with Proposition 1 implies the following statement.

Corollary The equivariant monodromy zeta function $\widetilde{\zeta}_{f}^{G}(t)$ is a rational function with coefficients in the Grothendieck ring $K_{0}$ (f.G-s.) of finite $G$-sets. 
Examples 1. Assume that the classical monodromy transformation $h=h_{f}: M_{f} \rightarrow$ $M_{f}$ is an element of the group $G$. This happens, in particular, when $f$ is a quasihomogeneous function and $G$ is its symmetry group. In this case the action of $h$ on $M_{f} / G$ is trivial and therefore $\widetilde{\zeta}_{f}^{G}(t)=(1-t)^{-\chi^{G}\left(M_{f}\right)}$. Thus in this case the equivariant monodromy zeta function is determined by the equivariant Euler characteristic $\chi^{G}\left(M_{f}\right)$ of the Milnor fibre $M_{f}$. This corresponds to the idea that in this situation the equivariant Euler characteristic $\chi^{G}\left(M_{f}\right)$ can be considered as an equivariant analogue of the monodromy zeta function: Ebeling and Gusein-Zade (2012a).

2. Let $f:\left(\mathbb{C}^{3}, 0\right) \rightarrow(\mathbb{C}, 0)$ be defined by $f(x, y, z)=x^{m}+y^{m}+z^{m}$. Consider the natural action of the group $\mathcal{S}_{3}$ of permutations on three elements on $\mathbb{C}^{3}$ by permutations of the coordinates. The function $f$ is $\mathcal{S}_{3}$-invariant. Blowing-up the origin, one gets a resolution of the function $f$. Let us assume that $m=6 k$. (In the other cases some of the strata $S_{m, H, \widehat{H}}$ below can be empty. These cases can be treated in the same way.) One has the following strata $S_{m, H, \widehat{H}}$ :

(a) $S_{m, \mathcal{S}_{3}, \mathcal{S}_{3}}$ consists of one point $P=(1: 1: 1) \in \mathbb{C P}^{2}=\pi^{-1}(0)$.

(b) $S_{m, \mathbb{Z}_{2}, \mathbb{Z}_{2}}$ consists of three lines $\{x=y\},\{x=z\}$ and $\{y=z\}$ (passing trough $P$ ) without their intersections with the strict transform of the surface $\{f=0\}$, i.e. with the curve $C=\left\{x^{m}+y^{m}+z^{m}=0\right\} \subset \mathbb{C P}^{2}$. One has

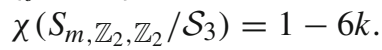

(c) $S_{m, \mathbb{Z}_{2},\langle e\rangle}$ consists of three points $(1:-1: 0),(1: 0:-1)$ and $(0: 1:-1)$. (For $6 \mid m$ these points do not lie on the curve $C$.) One has $\chi\left(S_{m, \mathbb{Z}_{2},\langle e\rangle} / \mathcal{S}_{3}\right)=1$.

(d) $S_{m, \mathbb{Z}_{3},\langle e\rangle}$ consists of two points $\left(1: \sigma: \sigma^{2}\right)$ and $\left(1: \sigma^{2}: \sigma\right)$, where $\sigma=$ $\exp (2 \pi i / 3)$. (For $6 \mid m$ these points do not lie on $C$.) One has $\chi\left(S_{m, \mathbb{Z}_{3},\langle e\rangle} / \mathcal{S}_{3}\right)$ $=1$.

(e) $S_{m,\langle e\rangle,\langle e\rangle}$ is the complement in $\mathbb{C P}^{2}$ to the curve $C$ and to all the strata above. One has $\chi\left(S_{m,\langle e\rangle,\langle e\rangle} / \mathcal{S}_{3}\right)=6 k^{2}-1$.

Now A'Campo formula (12) gives

$$
\begin{aligned}
\widetilde{\zeta}_{f}^{\mathcal{S}_{3}}(t)= & \left(1-t^{6 k}\right)^{-1} \cdot\left(1-t^{6 k}\right)^{(6 k-1)\left[\mathcal{S}_{3} / \mathbb{Z}_{2}\right]} \cdot\left(1-t^{3 k}\right)^{-\left[\mathcal{S}_{3} /\langle e\rangle\right]} \\
& \cdot\left(1-t^{2 k}\right)^{-\left[\mathcal{S}_{3} /\langle e\rangle\right]} \cdot\left(1-t^{6 k}\right)^{\left(1-6 k^{2}\right)\left[\mathcal{S}_{3} /\langle e\rangle\right]}
\end{aligned}
$$

\section{On Orbifold Versions of the Equivariant Monodromy Zeta Function}

For a $G$-variety $X$, its orbifold Euler characteristic $\chi^{\text {orb }}(X, G) \in \mathbb{Z}$ is defined, e.g., in Atiyah and Segal (1989) or Hirzebruch and Höfer (1990). For a subgroup $H$ of $G$, let $X^{H}=\{x \in X: H x=x\}$ be the fixed point set of $H$. The orbifold Euler characteristic $\chi^{\text {orb }}(X, G)$ of the $G$-space $X$ is defined, e.g., in Atiyah and Segal (1989) and Hirzebruch and Höfer (1990):

$$
\chi^{\text {orb }}(X, G)=\sum_{[g] \in \operatorname{Consub}(G)} \chi\left(X^{\langle g\rangle} / C_{G}(g)\right),
$$


where $C_{G}(g)=\left\{h \in G: h^{-1} g h=g\right\}$ is the centralizer of $g$, and $\langle g\rangle$ the subgroups generated by $g$.

There is a natural homomorphism of abelian groups $\Phi: K_{0}$ (f. $G$-s.) $\rightarrow \mathbb{Z}$ which sends the generator $[G / H]$ of $K_{0}$ (f. $G$-s.) to $\chi^{\text {orb }}(G / H, G)$ and therefore the equivariant Euler characteristic $\chi^{G}(X) \in K_{0}($ f. $G$-s. $)$ to the orbifold Euler characteristic $\chi^{\text {orb }}(X, G)$. For an abelian $G, \Phi([G / H])=|H|$ and $\Phi$ is a ring homomorphism, but this is not the case in general.

The Lefschetz number is a sort of generalization of the Euler characteristic: the Euler characteristic is the Lefschetz number of the identity map. The definition of the orbifold Euler characteristic gives the hint that there can be the corresponding definition(s) of the orbifold Lefschetz number of a $G$-equivariant transformation. It can be expressed through an equivariant version of the Lefschetz number with values in the Burnside ring: the image of the Lefschetz number by the homomorphism $\Phi$. The two versions $L^{G}(\varphi)$ and $\widetilde{L}^{G}(\varphi)$ of the equivariant Lefschetz number [see (4) and (6)] give two versions

$$
L^{\text {orb }}(\varphi)=\Phi\left(L^{G}(\varphi)\right) \text { and } \widetilde{L}^{\text {orb }}(\varphi)=\Phi\left(\widetilde{L}^{G}(\varphi)\right)
$$

of orbifold Lefschetz numbers.

The usual definition of the zeta function of a transformation [e.g. Eqs. (8), (9), (10) and (11)] gives two orbifold versions of the zeta function of a $G$-equivariant transformation $\varphi: X \rightarrow X$ :

$$
\zeta_{\varphi}^{o r b}(t)=\prod_{m \geq 1}\left(1-t^{m}\right)^{-s_{m}^{o r b} / m}, \text { and } \widetilde{\zeta}_{\varphi}^{o r b}(t)=\prod_{m \geq 1}\left(1-t^{m}\right)^{-\widetilde{s}_{m}^{o r b} / m}
$$

where $L^{\text {orb }}\left(\varphi^{m}\right)=\sum_{i \mid m} s_{i}^{\text {orb }}(\varphi)$ and $\widetilde{L}^{\text {orb }}\left(\varphi^{m}\right)=\sum_{i \mid m} \widetilde{s}_{i}^{\text {orb }}(\varphi)$.

The exponents $-\widetilde{s}_{m}^{\text {rrb }} / m$ are integers and therefore the orbifold monodromy zeta function $\widetilde{\zeta}_{\varphi}^{o r b}(t)$ is a rational function in $t$. The exponents $-s_{m}^{o r b} / m$ are in general rational numbers.

For instance, for $f$ from Example 2 in Sect. 5 one has

$$
\widetilde{\zeta}_{f}^{o r b}(t)=\left(1-t^{6 k}\right)^{-1+2(6 k-1)+\left(1-6 k^{2}\right)} \cdot\left(1-t^{3 k}\right)^{-1} \cdot\left(1-t^{2 k}\right)^{-1}
$$

This follows from the fact that, for $G=\mathcal{S}_{3}$ and for a subgroup $H$ of $\mathcal{S}_{3}$, $\chi^{\text {orb }}\left(\mathcal{S}_{3} / H, \mathcal{S}_{3}\right)=|H|$.

\section{References}

A'Campo, N.: La fonction zêta d'une monodromie. Comment. Math. Helv. 50, 233-248 (1975) Arnold, V.I., Gusein-Zade, S.M., Varchenko, A.N.: Singularities of Differentiable Maps. vol. II. Monodromy and Asymptotics of Integrals, Monographical Mathematics, vol. 83. Birkhäuser, Boston (1988)

Atiyah, M., Segal, G.: On equivariant Euler characteristics. J. Geom. Phys. 6(4), 671-677 (1989)

Gusein-Zade, S.M., Delgado, F., Campillo, A.: On the monodromy of a plane curve singularity and the Poincaré series of its ring of functions. Funktsional. Anal. i Prilozhen. 33(1), 66-68 (1999); translation in Funct. Anal. Appl. 33(1), 56-57 (1999) 
Campillo, A., Delgado, F., Gusein-Zade, S.M.: On Poincaré series of filtrations on equivariant functions of two variables. Mosc. Math. J. 7(2), 243-255 (2007)

Campillo, A., Delgado, F., Gusein-Zade, S.M.: Equivariant Poincaré series of filtrations. Rev. Mat. Complut. 26(1), 241-251 (2013)

Campillo, A., Delgado, F., Gusein-Zade, S.M.: An equivariant Poincaré series of filtrations and monodromy zeta functions. Rev. Mat. Complut. 28(2), 449-467 (2015)

Clemens, C.H.: Picard-Lefschetz theorem for families of nonsingular algebraic varieties acquiring ordinary singularities. Trans. Am. Math. Soc. 136, 93-108 (1969)

Crabb, M.C.: Equivariant fixed-point indices of iterated maps. J. Fixed Point Theory Appl. 2(2), 171-193 (2007)

Denef, J., Loeser, F.: Caractéristiques d'Euler-Poincaré, fonctions zêta locales et modifications analytiques. J. Am. Math. Soc. 5(4), 705-720 (1992)

Dzedzej, Z.: Fixed orbit index for equivariant maps. In: Proceedings of the Third World Congress of Nonlinear Analysts, Part 4 (Catania, 2000). Nonlinear Analytics, vol. 47(4), pp. 2835-2840 (2001)

Ebeling, W., Gusein-Zade, S.M.: Saito duality between Burnside rings for invertible polynomials. Bull. Lond. Math. Soc. 44, 814-822 (2012)

Ebeling, W., Gusein-Zade, S.M.: Equivariant Poincaré series and monodromy zeta functions of quasihomogeneous polynomials. Publ. Res. Inst. Math. Sci. 48(3), 653-660 (2012)

Gusein-Zade, S.M.:Integration with respect to the Euler characteristic and its applications. Uspekhi Mat. Nauk 65(393), 5-42 (2010) (no. 3); translation in Russ. Math. Surv. 65(3), 399-432 (2010)

Gusein-Zade, S.M.: On an equivariant analogue of the monodromy zeta function. Funktsional. Anal. i Prilozhen. 47(1), 17-25 (2013); translation in Funct. Anal. Appl. 47(1), 14-20 (2013)

Gusein-Zade, S.M., Luengo, I., Melle-Hernández, A.: Power structure over the Grothendieck ring of varieties and generating series of Hilbert schemes of points. Mich. Math. J. 54(2), 353-359 (2006)

Gusein-Zade, S.M., Luengo, I., Melle-Hernández, A.: An equivariant version of the monodromy zeta function. In: Geometry, Topology, and Mathematical Physics, pp. 139-146. American Mathematical Society Translation Series 2, 224, American Mathematical Society, Providence (2008)

Hirzebruch, F.: Höfer, Th: On the Euler number of an orbifold. Math. Ann. 286(1-3), 255-260 (1990)

Knutson, D.: $\lambda$-Rings and the Representation Theory of the Symmetric Group. Lecture Notes in Mathematics, vol. 308. Springer, Berlin, New York (1973)

Lück, W., Rosenberg, J.: The equivariant Lefschetz fixed point theorem for proper cocompact $G$-manifolds. In: Farrell, F.T., Lück, W. (eds.) High-Dimensional Manifold Topology, pp. 322-361. World Science Publishing, River Edge (2003)

tom Dieck, T.: Transformation Groups and Representation Theory. Lecture Notes in Mathematics, vol. 766. Springer, Berlin (1979)

Verdier, J.-L.: Caractéristique d'Euler-Poincaré. Bull. Soc. Math. France 101, 441-445 (1973)

Wall, C.T.C.: A note on symmetry of singularities. Bull. Lond. Math. Soc. 12(3), 169-175 (1980) 